\title{
Injerto dermo-epidérmico como tratamiento del siliconoma de extremidad inferior
}

\author{
Juan Pablo Camacho' ${ }^{1}$, Manuel Quiroz, Rodrigo Subiabre ${ }^{2}$ y Wilfredo Calderón ${ }^{2,3}$
}

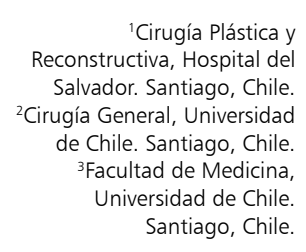

Recibido el 2 de noviembre de 2016, aceptado el 9 de enero de 2017.

Correspondencia a: Manuel Quiroz quirozflores.manuel@gmail.

\section{Dermoepidermal graft as treatment of lower extremity siliconoma}

Introduction: Despite the evidence of poor performance and risks of using silicone subcutaneous injections to improve body contour, this technique is still used by untrained people, generating an iatrogenic entity with serious complications. Case report: 56 year old female presented left lower extremity cellulitis. A history of previous intervention with silicone injections in face, back of hands, buttocks and legs in year 2000 was obtained. An inflammatory process progressed locally and systemically with multiple abscesses, subcutaneous thickness in the entire leg and sepsis, requiring multiple surgical procedures, reaching fascial and muscle planes exposition of the left leg. The patient underwent local therapy with negative pressure systems and subsequent dermoepidermal graft. Multidisciplinary management was performed on a critical unit. The results were satisfactory, preserving the limb with full coverage. She was discharged after four months of inpatient management. Conclusion: Despite knowing the risks of silicone injection (industrial or medical) it remains a common procedure. In our patient she presented as leg fasciitis, requiring aggressive surgical treatment. Knowing this entity, its diagnosis and treatment is essential to treat patients with serious complications.

Key words: Liquid silicone injection; Siliconoma; Ulcer.

\section{Resumen}

Introducción: A pesar de la evidencia sobre los malos resultados y riesgos del uso de inyecciones subcutáneas de silicona para mejorar el contorno corporal, esta técnica sigue siendo utilizada por personas no calificadas. Caso clínico: Paciente de 56 años quien consultó por cuadro de celulitis en pierna izquierda. Se obtuvo el antecedente de intervención con silicona en cara, dorso de las manos, glúteos y piernas el año 2000. El cuadro progresó con múltiples abscesos en la totalidad de la pierna y sepsis. Se realizaron aseos quirúrgicos exponiendo planos fascial y muscular, se utilizó terapia local con sistemas de presión negativa y posterior injerto dermo-epidérmico (IDE). El manejo multidisciplinario se realizó en unidades críticas. Los resultados del manejo fueron satisfactorios, salvando la extremidad con cobertura total, logrando el alta luego de 4 meses. Conclusión: A pesar de conocerse los peligros de la inyección de silicona, este sigue siendo un procedimiento frecuente. En nuestra paciente se manifestó como fascitis de la pierna, requiriendo tratamiento quirúrgico agresivo. Conocer esta entidad, su diagnóstico y tratamiento es fundamental para tratar pacientes con complicaciones graves.

Palabras clave: Inyección de silicona líquida; Siliconoma; Úlcera.

\section{Introducción}

El uso de sustancias para modificar el contorno corporal tiene una historia extensa, durante la cual se han desarrollado diferentes técnicas y materiales.

Las siliconas llamaron la atención desde su descubrimiento por poseer propiedades ventajosas sobre otras sustancias sintetizadas. La silicona de uso médico es inerte, en dosis bajas no migra, su efecto es permanente y puede prepararse desde líquida hasta sólida ${ }^{1,2}$.

Durante mediados del siglo XX se desarrollaron múltiples aplicaciones, sin embargo, con el paso de los años se reportaron complicaciones de distinta magnitud $^{3,4}$.

Las siliconas de uso industrial, llamadas siloxa- 
nos polimerizados, de fórmula química ( $\mathrm{R} 2 \mathrm{SiO}$ ) $\mathrm{n}$, donde $\mathrm{R}$ es un grupo orgánico, (metilo, etilo o fenilo) y una cadena inorgánica de silicio y oxígeno (-Si-O-Si-O-Si-O-), con grupos laterales orgánicos unidos a los átomos de silicio. El siloxano más común es el polidimetilsiloxano lineal (PDMS), un aceite de silicona.

En relación con sus características, es una sustancia biológicamente inerte, de baja toxicidad, térmica y químicamente estable, es resistente a la luz ultravioleta y al oxígeno, su uso habitual es como sellante y lubricante.

Los pacientes sometidos a tratamientos cosméticos con sustancias de relleno realizados por personas no calificadas, la mayoría de las veces desconocen la sustancia que se ha utilizado y la forma de preservación que esta ha tenido, por lo tanto, los riesgos que derivan de esta práctica son diversos.

El siliconoma es una reacción granulomatosa por cuerpo extraño que ocurre como resultado del implante subcutáneo de silicona. Las manifestaciones clínicas generalmente se presentan en un período variable de meses a años posterior al procedimiento, pudiendo causar ulceración y necrosis de los tejidos.

Es más frecuente en el género femenino, que representa el 97\% de los casos, y la característica común es la realización de un procedimiento estético por personal no calificado con sustancias desconocidas $^{1-6}$.

El tratamiento de esta entidad es multidisciplinar, el manejo médico es importante para tratar el cuadro inflamatorio e infeccioso y la cirugía es necesaria para remover los granulomas, la silicona y el tejido desvitalizado ${ }^{1,2}$.

En casos graves la alternativa quirúrgica no es de elección y hay escasa evidencia que señale la utilidad del injerto dermo-epidérmico (IDE) como alternativa terapéutica en estos casos.

Presentamos la experiencia en nuestro servicio en el manejo de un caso.

\section{Presentación del caso}

Mujer de 56 años, con antecedentes de tabaquismo crónico, estenosis crítica de la arteria ilíaca común derecha tratada mediante plastía con balón y stent el año 2013.

La paciente consultó en agosto de 2015 por cuadro de 4 días de evolución de aumento de volumen y eritema en la extremidad inferior izquierda, asociado a fiebre de hasta $40{ }^{\circ} \mathrm{C}$. Al examen presentó en extremidad inferior izquierda: úlcera maleolar medial con secreción purulenta en uno de sus bordes, erite- ma desde la rodilla a distal y calor local; se sospechó una celulitis por mordedura de insecto, a pesar de no contar con el antecedente claro, sin embargo, el cuadro inició con dolor urente, localizado en la cara medial de la extremidad inferior.

Debido al gran compromiso de la extremidad en una paciente sin comorbilidades descompensadas, se preguntó por otros antecedentes y se obtuvo el dato de una intervención de inyección de silicona industrial en frente, manos y extremidad inferior izquierda el año 2000 (Figura 1).

Se realizó aseo quirúrgico identificando celulitis, múltiples abscesos y fístulas de silicona en la extremidad, cubriendo el 3\% de la superficie corporal total, con gran reacción granulomatosa asociada. Se realizó escarectomía tangencial, se enviaron muestras para biopsia y cultivos e iniciamos antibióticos de forma empírica (Figura 2).

El informe histopatológico señaló piel y tejido subcutáneo con proceso inflamatorio crónico y agudo supurado, asociado a áreas granulomatosas de tipo cuerpo extraño compatible con siliconoma.

El control imagenológico de las extremidades inferiores se hizo mediante tomografía computarizada (TC) con contraste, que evidenció inicialmente zonas de respuesta inflamatoria crónica y zonas abscedadas; a su vez se encontró indemnidad de la circulación arterial, la cual se verificó con ecografía Doppler.

Se realizaron múltiples aseos quirúrgicos y manejo local con terapia de presión negativa semanal y bisemanal.

Los cultivos de tejido mostraron crecimiento de Pseudomonas aeruginosa resistente a carbapenémicos, por lo que se ajustó el tratamiento antibiótico y se asoció a curaciones con povidona yodada.

Debido al dolor intenso (10/10 según escala visual análoga) durante gran parte del día, se realizó manejo multimodal, AINE intravenosos, acetaminofén oral y opioides en infusión continua.

Se discutió en reunión de equipo quirúrgico y con la familia la eventual necesidad de amputación de la extremidad inferior izquierda.

La paciente evolucionó con deterioro de la función renal de causa multifactorial, requiriendo 2 sesiones de diálisis.

Con la paciente en condiciones estables se decidió realizar IDE en la cara anterior de la pierna izquierda y evaluar la respuesta.

Debido a la buena respuesta con prendimiento adecuado del injerto se decidió realizar cobertura con IDE de la extremidad mediante intervenciones consecutivas, evolucionando sin complicaciones locales (Figura 3). 


\section{CASO CLÍNICO}

Figura 1. Presentación inicial, eritema difuso y zonas fluctuantes en pierna izquierda.

Figura 2. Resultado luego de múltiples aseos quirúrgicos, eliminando la totalidad del tejido comprometido por la silicona.

Figura 3. Primera cobertura mediante IDE de muslo bilateral, logrando un prendimiento cercano al $100 \%$.
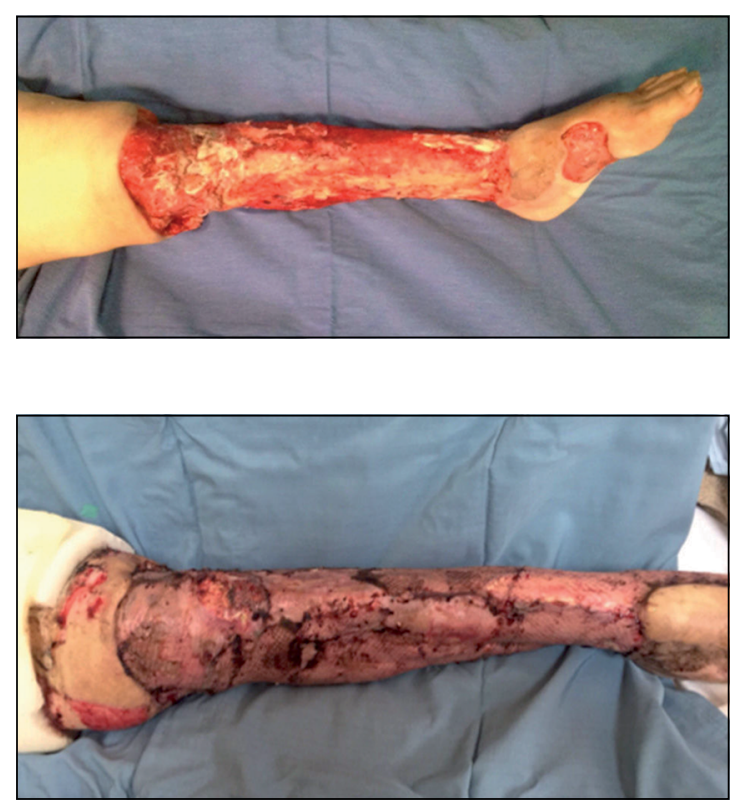

Una vez completado el tratamiento antibiótico y normalizados los parámetros nutricionales, se indicó el alta luego de 4 meses de hospitalización.

La paciente fue derivada a fisioterapia para rehabilitación de la extremidad y se controló de forma ambulatoria en policlínico, encontrándose en buenas condiciones, sin signos inflamatorios en la extremidad y con movilidad y fuerza conservadas; la evolución de los injertos se observó a los 2 meses del alta (Figura 4).

\section{Discusión}

A pesar del reporte de los devastadores resultados que ocasiona el uso de silicona industrial en procedimientos estéticos, siguen reportándose casos en todo el mundo.

Las características de la silicona de uso médico difieren de las siliconas utilizadas en procedimientos de carácter clandestino, ya que no poseen estándares de purificación, además, debido a la gran cantidad de sustancias empleadas y al desconocimiento general por parte del paciente, el dato de un procedimiento cosmético con inyecciones parece ser el más relevante para establecer la sospecha ${ }^{5}$.

El siliconoma se ha descrito en el género femenino entre un 79 y un $97 \%$ de los casos. Sin embargo, ha aumentado la incidencia de esta entidad en hombres transgénero ${ }^{1,6-8}$.

Se desconoce una respuesta antigénica específica a la silicona líquida; es frecuente la respuesta inflamatoria y la formación de granulomas, lo que puede exacerbarse en presencia de un cuadro infeccioso local como en nuestra paciente ${ }^{5,9-11}$.

El cuadro clínico presentado se manifestó 15 años después del procedimiento y la forma de presentación fue aguda, caracterizada por eritema, edema, dolor, reacción inflamatoria tipo granuloma, ulceración y necrosis de la piel. El compromiso sistémico estuvo dado por fiebre, astenia y malestar general. Lo anterior es compatible con lo reportado sobre la latencia de este cuadro, describiéndose hasta 25 años después del procedimiento ${ }^{1,7,10-13}$.

El estudio imagenológico lo realizamos con TC con contraste intravenoso, permitiendo identificar áreas de inflamación y colecciones en la extremidad; el uso de resonancia magnética (RM) sería superior para delimitar el compromiso de los tejidos circundantes. En pacientes añosos es relevante estudiar el compromiso vascular aterosclerótico como patología asociada al cuadro.

La anatomía patológica, generalmente, describe una inflamación crónica con reacción a cuerpo extraño y en algunos casos calcificación distrófica ${ }^{1}$.
Figura 4. Al control en policlínico, presenta cobertura total y funcionalidad. 
Con relación al tratamiento médico, en nuestro caso el uso de AINE y opioides (tramadol) fue fundamental para lograr un manejo adecuado del dolor, sin embargo, presentamos una depresión respiratoria severa debido a su uso en infusión continua, por lo que modificamos su indicación. El uso de corticoides está descrito en cuadros de inflamación local sin ulceración, ya que retardarían la cicatrización en estos casos.

Como manejo farmacológico se han utilizado medicamentos inmunomoduladores como imiquimod, tacrolimus, pimecrolimus, etanercept y alopurinol e isotretinoína con resultados variables.

Respecto a la cirugía, la evidencia proviene de reportes clínicos y series pequeñas, en las que se opta por diferentes procedimientos acordes al problema clínico planteado.

Se conocen experiencias con dermolipectomía, técnicas de aspiración modificada y resección local, entre otras ${ }^{13}$.

La resección quirúrgica es la alternativa más frecuente, consiste en un aseo amplio delimitando y resecando las zonas de infiltración por silicona. Hay que tener en consideración que el procedimiento puede generar migración de silicona o no lograr removerla completamente ${ }^{13,14}$.

En pacientes graves las alternativas quirúrgicas no están indicadas, sino más bien el manejo conservador y la estabilización; es posible considerar, en casos como el nuestro, ante el compromiso de extremidades, la necesidad de amputación ${ }^{13,15}$

El aseo quirúrgico y la resección del tejido infiltrado, limitar la infección en caso de encontrarla y considerar los cuidados locales del lecho serían un pilar en casos que requieren múltiples aseos.

Las terapias de presión negativa se han utilizado en esta entidad por sus beneficios conocidos; en nuestro caso este tipo de terapia fue útil, sin embargo, presentó complicaciones descritas como la mala tolerancia y dolor de difícil manejo ${ }^{13}$.

Las técnicas de cobertura son diversas y responden a la necesidad, extensión del compromiso y ubicación. Existe escasa evidencia sobre el uso del IDE como cobertura; en nuestro caso, dada la extensión del área comprometida, la gravedad de la paciente, así como la necesidad de una cobertura pronta y funcional nos inclinó por esta alternativa, logrando buenos resultados.

El manejo de las variables psicoafectivas y el adecuado control del dolor fueron fundamentales en nuestro caso y recomendamos considerarlas al tratar esta entidad.

Es importante mencionar que en este caso tratamos la extremidad inferior izquierda, que manifestó el cuadro clínico luego de 15 años de latencia, sin embargo, la paciente también presenta silicona en la extremidad inferior derecha, en la cara y en las manos, así pues, la posibilidad de repetir el cuadro es alta. Se han reportado casos con latencia de hasta 25 años, por lo tanto, el tiempo no es un factor que disminuya el riesgo.

Consideramos que debe realizarse seguimiento clínico y ante la sospecha del cuadro evaluar el área afectada con RM o TC, en última instancia realizar una biopsia quirúrgica, para tratar el cuadro en forma precoz.

El siliconoma es una entidad que debe considerarse en pacientes que se someten a procedimientos estéticos no regulados. Su tratamiento debe ser multidisciplinario. Es primordial la cirugía de aseo, considerar el posterior IDE como una alternativa válida de cobertura.

\section{Responsabilidades éticas}

Protección de personas y animales. Los autores declaran que para esta investigación no se han realizado experimentos en seres humanos ni en animales.

Confidencialidad de los datos. Los autores declaran que han seguido los protocolos de $\mathrm{su}$ centro de trabajo sobre la publicación de datos de pacientes.

Derecho a la privacidad y consentimiento informado. Los autores han obtenido el consentimiento informado de los pacientes y/o sujetos referidos en el artículo. Este documento obra en poder del autor de correspondencia.

\section{Financiación}

El presente trabajo no posee fuente de financiación.

\section{Conflicto de intereses}

Los autores declaran no tener ningún conflicto de intereses. 


\section{CASO CLÍNICO}

\section{Bibliografía}

1. Coiffman F. Alogenosis iatrogénica. Una nueva enfermedad. Cir Plast Iberolatinoam. 2008;34:1-10.

2. Narins RS, Beer K. Liquid injectable silicone: A review of its history, inmunology, technical considerations, complications, and potential. Plast Reconstr Surg. 2006;118:77-84.

3. Chasan P. The history of injectable silicone fluids for soft-tissue augmentation. Plast Reconstr Surg. 2007;120:2034-40.

4. Ashinoff R. Oveview: Soft tissue augmentation. Clin Plast Surg. 2000;27:479-89.

5. Chiu ES, Sharma S, Siebert JW. Salvage of silicone-treated facial deformities using autologous free tissue transfer. Plast Reconstr Surg. 2005;116:1195-203.
6. Mello D, Gonçalves K, Fraga M, Perin L, Helene A Jr. Local complications after industrial liquid silicone injection case series. Rev Col Bras Cir. 2013;40:37. 42.

7. Wilson E, Rapues J, Jin H, Raymond F. The use and correlates of illicit silicone or fillers in a population-based sample of transwomen, San Francisco. J Sex Med. 2014;11:1717-24.

8. Prather C, Jones D. Liquid injectable silicone for soft tissue augmentation. Dermatol Ther. 2006;19:159-68.

9. Rapaport MJ, Vinnik C, Zarem H. Injectable silicone: Cause of facial nodules, cellulitis, ulceration, and migration. Aesthetic Plast Surg. 1996;20:267-76.

10. Carella S, Romanzi A, Ciotti M, Onesti MG. Skin Ulcer: A longterm complication after massive liquid silicone oil infiltration. Aesth Plast Surg. 2013;3:1220-2.

11. Dragu A, Theegarten D, Bach A, Polykandriotis E, Arkudas A, Kneser $\mathrm{U}$, et al. Intrapulmonary and cutaneous siliconomas after silent silicone breast implant failure. Breast J. 2009;15:496-9.

12. Ellis A, Cohen J, High W. Granulomatous reaction to silicone injection. J Clin Aesthet Dermatol. 2012;5:44-53.

13. Loustau HD, Mayer HF, Catterino L. Dermolipectomy of the thighs and buttocks to solve a massive silicone oil injection. Aesthetic Plast Surg. 2009;33:657-60.

14. Gutiérrez L, Montes A. Siliconomas. Caso clínico. Rev Med Chile. 2002;130:793-7.

15. Fontbona M, Altura M, Gacitúa $\mathrm{H}$, Britzmann B. Consecuencias de la inyección de silicona líquida en mamas. Rev Chi Cir. 2003;55:389-93. 